\title{
A Semantic Distances-Based Approach for a Deeply Indexing of Learning Objects
}

\author{
https://doi.org/10.3991/ijet.v14i06.9738
}

\author{
Kamal El Guemmat ${ }^{(凶)}$, Sara Ouahabi \\ Hassan II University, Casablanca, Morocco \\ k.elguemmat@gmail.com
}

\begin{abstract}
Educational search engines are important for users to find learning objects (LO). However, these engines have not reached maturity in terms of searching, they suffer from several worries like the deep extraction of notions which diminishes their performance. The purpose of this paper is to propose a new approach that allows depth extraction of LO's notions to increase the relevance level of educational search engines.

The proposed approach focuses on semi-automatic indexing of textual LO and more precisely the deeper relations of sentences that flesh out explanations. It based on linguistic structures and semantic distances between specific and generic notions according to OntOAlgO ontology. The notions obtained will be improved by learning object metadata (LOM) and will be represented semantically in final index.

The tests performed on algorithmic LO, proving the usefulness of our approach to educational search engines. It increases the degree of precision and recall of notions extracted from LO.
\end{abstract}

Keywords - Educational search engine, Learning object, Linguistic structure, Semantic distance, Ontology, Learning object metadata.

\section{$1 \quad$ Introduction}

The search of information dates back to 1948 [1] it is a process of storage, organization, representation, and access to information by the user.

These engines are used in several fields. In the field of teaching and research, they are used by e-learning systems, they are called educational search engines [2].

Educational search engines bring a lot of benefits to e-learning systems; as the identification, adaptation, reuse and sharing of learning objects (LO).

Educational search engines are based on the exploratory learning approach allowing the learner to direct his own study according to his search and allowing the teacher to deposit and index his course.

However, these search engines encounter a primary concern to the exact identification of LO. The degree of satisfaction is still far from the expectations of users. 
The presented approach adopts the one that was developed by authors in previous work, it is the multilayer indexing model of teaching and learning resources (MIMTLR) [3] to extract deeply educational search engines notions and increase the degree of precision and recall.

The proposed approach is based on the principle of search engine, where there is the principle of indexing and searching. These principles make it possible to recover resources concerned from a huge database. This is usually achieved by the indexing and searching techniques either by metadata, classical or semantic techniques.

Since 1948, the date of the appearance of the search engines and until today several researches have resulted considerable developments in all search engines fields. In the case of e-learning there are several important works [4], [5], [6], [7], [8], [9], [10], [11], [12], [13], [14], [15], [16], most of them adopt metadata, classical, and semantic indexing techniques, where there are some gaps sometimes.

The metadata indexes only the fields predefined by the standard and the classical techniques extract only nonsensical terms, however these techniques remain nonexhaustive, and offer no contextual semantic representation of the content.

The semantic techniques index only notions related to specific use, that is to say a notion and its exact use in the field, for example in the domain of algorithmic there is the variable notion and its boolean use. These notions will be taken without interest in generic uses of a particular domain (definition, use, exercise, corrected, example).

The techniques that combine semantic indexing with metadata do not include the notions and their generic and specific use to metadata in an ontology.

However, there is no semi-automatic techniques, which makes it possible to extract via a LO the metadata and the notions related to a specific and generic use of a particular domain.

Yet, there is a work to remedy these worries [3], which is based on metadata and semantic indexing according to OntOAlgO ontology, which allows to index the notions while considering the specific and generic uses of a particular domain. This technique is based on linguistic structures, despite that, it itself suffers from several problems. There is no deep extraction of notions. It just extracts the notions of the same explanatory sentence and their generic uses.

The purpose of this article is to improve this technique by taking into consideration deeper relations in sentences that flesh out explanations and ensure that they convey meaning between them. It based on linguistic structures and semantic distances between notions according to OntOAlgO ontology.

To calculate the semantic distance several measures have been adopted as, the relation of the same hierarchical level was used, the subsumption relation is-a and the measurement of [17].

The notions obtained will be improved by the learning object metadata (LOM) and will be represented semantically in the index.

The proposed approach, and after a validation test, it results a good result. The goal is reached; there is an increase in the degree of precision and recall of LO notions.

The rest of this paper will be distributed as follows, the following section presents the review of related works, search engine in general, and educational search engines, 
this section will be followed by the proposed approach its validation and finally the conclusions and perspectives.

\section{Review of Related Works}

\subsection{Search engine}

Several definitions of information retrieval have been defined describe searching as a process of storage, organization, representation, and access to information by the user.

Before providing to users the right results, the information retrieval goes through several stages as described by R.K. Belew in his book "FOA- Finding Out About" [18].

R.K. Belew proposed a process that now sees itself as a general process of information retrieval.

The general process is divided into several parts of which three processes are essential, indexing process, search process and evaluation of the answer.

Indexing must support certain issues, either it's interested in recovering important terms in a document with their weights what is called classic indexing or is interested in recovering the meaning of the important words of a document, relations and events that link them, this type of indexing is called semantic [19].

The next section presents the educational search engines that are based on the techniques mentioned before.

\subsection{Educational search engines}

General search cannot and probably should not meet the specific needs of disciplines [20], the need for improvement is a big challenge in this case. Online learning systems interact with educational applications of search engines to overcome the limitations of general search, E-learning-based search engines offer many advantages.

Like generic search engines, the educational ones are based on indexing and search techniques. There are four indexing and searching techniques. There is the technique of classical, sematic indexing and searching.

There is another type of indexing by metadata that does not fit in the content of the LO but just indexes its structure, it is done most of the time manually by human beings, it is based on standards for example the LOM; confirming the usefulness of LO searching [21].

The hybrid technique [5], it is a combination of different techniques that becomes a necessity to take advantage of its advantages and to include them in a powerful model that can meet the indexing requirements of an LO.

In parallel with the indexing and searching stage, there is another important step in evaluating and improving of search; like the type of search [22], the ranking of the 
results [15], [12], query enrichment [8], profile [23], [24], [25], LO language and implementation platform.

There are several educational search engines adopting indexing and searching techniques for the LO case. In the following will be presented the MIMTLR search engine [3] on which our work is focused.

It adopts the hybrid technique and more precisely metadata and semantic indexing; the disambiguation is by linguistic structures and ontology.

This search engine is a prototype that allows to find the textual algorithmic LO in French language. MIMTLR is inspired by the general architecture of an information retrieval system, there are two processes (indexing and searching process).

Indexing is hybrid, based on semantic and metadata technique. Disambiguation considers two layers (Layer1: Structure of the object, Layer2: Semantic content of the object) to generate an index that represents the characteristics of the LO enriched by the semantics of the content. MIMTLR indexes LO through ontologies and algorithms for automatic of natural language processing (NLP). Disambiguation goes through two steps, Step 1, Indexer manually indexes the technical part of the LO (manual indexing), Step 2, the teacher indexes semi automatically LO according to its content, it is based on an ontology and patterns of extraction.

In step 1, indexer will manually index the technical part of LO. The choice is opted for manual indexing, because the LOM standard has most fields that had to be deduced by the human being.

Since the manual model has a major disadvantage, which causes incoherent and subjective indexing, the fact that the same document can be indexed by different methods according to the indexer will be difficult to apply on a large corpus. In addition, the main purpose of the LOM is the interoperability of the indexes. For all these reasons the indexer makes his choice since a several suggestions from a controlled vocabulary.

Step 2 allows to index the notions while taking into consideration the notions related to a specific and generic uses of a particular domain. In this step there will be detected several types of notion. Notion of application (NoA), are the key concepts that model the LO. Notion of generic use (NoUG), are the notion independent of a particular domain, such as definition, Example, Exercise, etc. Notion of specific use (NoUS), are the notion specific for each NoA, for example in algorithmic domain there is for the NoA Variable the NoUS Boolean, Numeric, etc.

The detection of NoA and NoUS, NoUG passes by the segmentation of the LO in sentences, the idea of segmentation of LO comes from a grammatical study of the French language.

At the level of the French language, there are several textual sequences (narration, description, explanation, argumentation, etc.).

An explanatory passage is rather didactic, having recourse to facilitate understanding, there are two types of sentence, explanatory and linguistic.

These are a set of means that allow to formulate a clear explanation, there are different explanatory sentences (The definition, the example, the visual illustration, etc.). 
As far as, some linguistic phrases flesh out explanations (reported speech, subordination, footnotes, phrases with special construction).

MIMTLR treats the LO, while segmenting the LO in sentences, it finds in each sentence the NoA before passing to the NoU from OntOAlgO reference ontology, the patterns of extraction adopted for detection are as follows:

- NoA [Ni] * NoUGx [UGj]*, $\mathrm{x}$ belongs to $\{1 \ldots, 5\}$; i et $\mathrm{j}$ belongs to $\{1 \ldots, \mathrm{n}\}$.

- NoUGx [UGi] * NoA $[\mathrm{Nj}]^{*}, \mathrm{x}$ belongs to $\{1 \ldots, 5\}$; i et $\mathrm{j}$ belongs to $\{1 \ldots, \mathrm{n}\}$.

- NoUS [USi] * NoUGx [UGj]*, $\mathrm{x}$ belongs to $\{1 \ldots, 5\}$; i et $\mathrm{j}$ belongs to $\{1 \ldots, \mathrm{n}\}$.

- NoUGx [UGi] $*$ NoUS [USj] ${ }^{*}$, $x$ belongs to $\{1 \ldots, 5\}$; i et $\mathrm{j}$ belongs to $\{1 \ldots, \mathrm{n}\}$.

Knowing that: "*" is a wildcard, that is, a sequence of terms.

For the notions of applications are collected in a table named NoA [Ni] where $\mathrm{Ni}$ is a notion with $\mathrm{i}$ belongs to $\{1 \ldots, \mathrm{n}\}$. These notions are defined in the first level of the conceptual hierarchical tree of the ontology. Here is an example of OntOAlgO NoA [variable, loop, function, etc.].

For the notions of specific use are the rest of the levels of the hierarchical tree of the ontology. These notions are gathered in a table named NoUS [USi] where USi is a notion with i belongs to $\{1 \ldots, \mathrm{n}\}$. Here is an example of OntOAlgO NoUS [numeric operators, alphanumeric operators, Boolean operators, etc.].

Then the indexer indicates the possible values for the NoUG, NoUGx [UGi], where $\mathrm{x}$ belongs to $\{1 \ldots, 5\}$ and $\mathrm{UGi}$ is a descriptor with $\mathrm{i}$ belongs to $\{1 \ldots, \mathrm{n}\}$. Here is an example for the NoUG1 [is, we define, definition, etc.] indicates the set of descriptors that reflects NoUG1. The NoUG1 (for definition). NoUG2 (for use). NoUG3 (for exercise). NoUG4 (the correction). NoUG5 (for example).

At the end of this step, the index will be represented according to an ontology in OWL language.

The searching is a comparison between the LO and the textual query that is written in a free language that will be enriched by other terms (synonym) and translated according to the SPARQL language, before launching the comparison, there is a rule which will be applied on the request of the user. MIMTLR processes the request to filter the notions, what remains will be considered as LOM information. The application returns the URL of the LO defined in layer 1, with other information from the layer 1 as additional information on the LO. The results are not ranked according to their order of relevance. The user's profile is not taken into consideration to customize searches. The implementation is done by the J2EE platform, Jena API, RDF language and the PROTEGE tool.

In this study, MIMTLR based on metadata and semantic indexing, despite that, it itself suffers from several problems. There is no deep extraction of notions. It just extracts the notions of the same explanatory sentence with their generic uses.

The approach that will be presented in what follows improve this technique by taking into consideration deeper relations in sentences that flesh out explanations and ensure that they convey meaning between them. 


\section{$3 \quad$ Methodology}

After all that was presented; there is a huge lack in terms of deep extraction of the notions related to a specific and generic uses of a particular domain so gathered them together with metadata in an ontology semi-automatically.

So, the present approach will improve the existing approach [3]. This approach is chosen after a comparison with the other approaches and it was noted that the latter is remarkable in its field. It is based on a semi-automatic metadata, semantic indexing with the same ontological representation.

This approach marks for the moment just the notions of the same sentence; the relation between NoA and NoUG or between NoUS and NoUG.

The new approach takes care of all this with the addition of a deeper notion extraction module to identify the relation between the NoA and NoUS or between the NoA from the sentences filling explanations.

The extracted notions will be improved by the LOM schema so that the index is richer.

\subsection{Process of the approach}

The process followed by the approach is as follows cf. figure 1 .

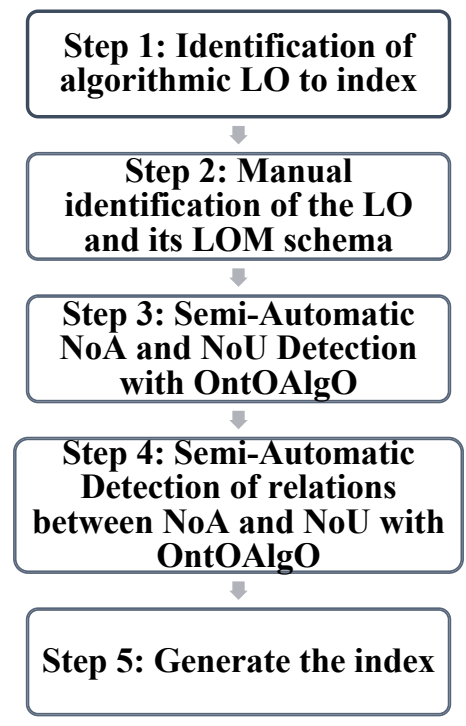

Fig. 1. Process of the approach

The following sub-sections details the different stages.

Step 1: Identification of algorithmic LO to index: At this step, which indicates the LO to be indexed, the proposed approach only supports textual LO 
Step 2: Manual identification of the LO and its LOM schema: The indexer makes his choice since a several suggestions from a controlled vocabulary. Once the fields are indicated, they will be populated in the concepts defined in the first layer of MIMTLR.

Step 3: Semi-Automatic NoA and NoU Detection with OntOAIgO

Semi-automatic detection of NoA, NoUS and their NoUG in sentences: These sentences are already exploited in the approach of [3] to extract the NoA and NoU as well as the relation between them.

The shapes or patterns of extraction adopted for detection are as follows:

- NoA [Ni] $*$ NoUGx [UGj]*, $x$ belongs to $\{1 \ldots, 5\}$; i et $j$ belongs to $\{1 \ldots, n\}$.

- NoUGx [UGi] * NoA $[\mathrm{Nj}]^{*}, \mathrm{x}$ belongs to $\{1 \ldots, 5\}$; i et $\mathrm{j}$ belongs to $\{1 \ldots, \mathrm{n}\}$.

- NoUS [USi] * NoUGx [UGj]*, x belongs to $\{1 \ldots, 5\}$; i et $\mathrm{j}$ belongs to $\{1 \ldots, \mathrm{n}\}$.

- NoUGx [UGi] * NoUS [USj]*, $x$ belongs to $\{1 \ldots, 5\}$; i et $\mathrm{j}$ belongs to $\{1 \ldots, \mathrm{n}\}$.

In what follows will be added other deeper patterns between the notions of the sentence to enrich it.

Semi-automatic detection of NoA and NoUS and their relations in sentences: From the analyzes that have been investigated on explanatory sentences, other relations have been detected manually between the notions as follows: NoA-NoUS or NoUS-NoA or NoA-NoA or NoUS-NoUS.

As a result, the approach automates the semantic detection. The shapes or patterns of extraction adopted for detection are as follows:

- NoUS [USi] * NoUS [USj]*, i and j belongs to $\{1 \ldots, \mathrm{n}\}$.

- NoA $[\mathrm{Ni}] * \operatorname{NoA}[\mathrm{Nj}]^{*}, \mathrm{i}$ and $\mathrm{j}$ belongs to $\{1 \ldots, \mathrm{n}\}$.

- NoUS [USi] * NoA $[\mathrm{Nj}]^{*}, \mathrm{i}$ and $\mathrm{j}$ belongs to $\{1 \ldots, \mathrm{n}\}$.

- NoA $[\mathrm{Ni}] *$ NoUS [USj] ${ }^{*}, \mathrm{i}$ and $\mathrm{j}$ belongs to $\{1 \ldots, \mathrm{n}\}$.

To take into consideration a such detection, a semantic relation between these notions must exist as will be explained in the next section.

Step 4: Semi-automatic detection of relations between NoA and NoUS

The indexing approach identifies the semantic relation between NoA-NoUS or NoUS-NoA NoA-NoA or NoUS-NoUS.

For that, it was used the semantic distance between the notions of the sentence in order to deduce the relation between them. The relation of the same hierarchical level was used, the subsumption relation is-a and the measurement of [17] which showed better correlation coefficients [26].

The following formula defines the measurement of $\mathrm{Wu}$ and Palmer:

$\operatorname{Sim}(\mathrm{C} 1, \mathrm{C} 2)=\frac{2 * N 3}{N 1+N 2}(1)$

This similarity is based on the following principle. Let $\mathrm{C} 1$ and $\mathrm{C} 2$ be two elements of an ontology formed by a set of nodes and a root node $\mathrm{R}$. The principle of similarity calculation is based on the distances ( $\mathrm{N} 1$ and $\mathrm{N} 2)$ that separate the nodes $\mathrm{C} 1$ and $\mathrm{C} 2$ from the root node and the distance $\mathrm{N} 3$ that separates the subsuming concept $\mathrm{C} 3$ of $\mathrm{C} 1$ and $\mathrm{C} 2$ from the root node. 
The hierarchical tree of OntOAlgO will be as follows cf. Figure 2 to be in harmony with the proposed measures:

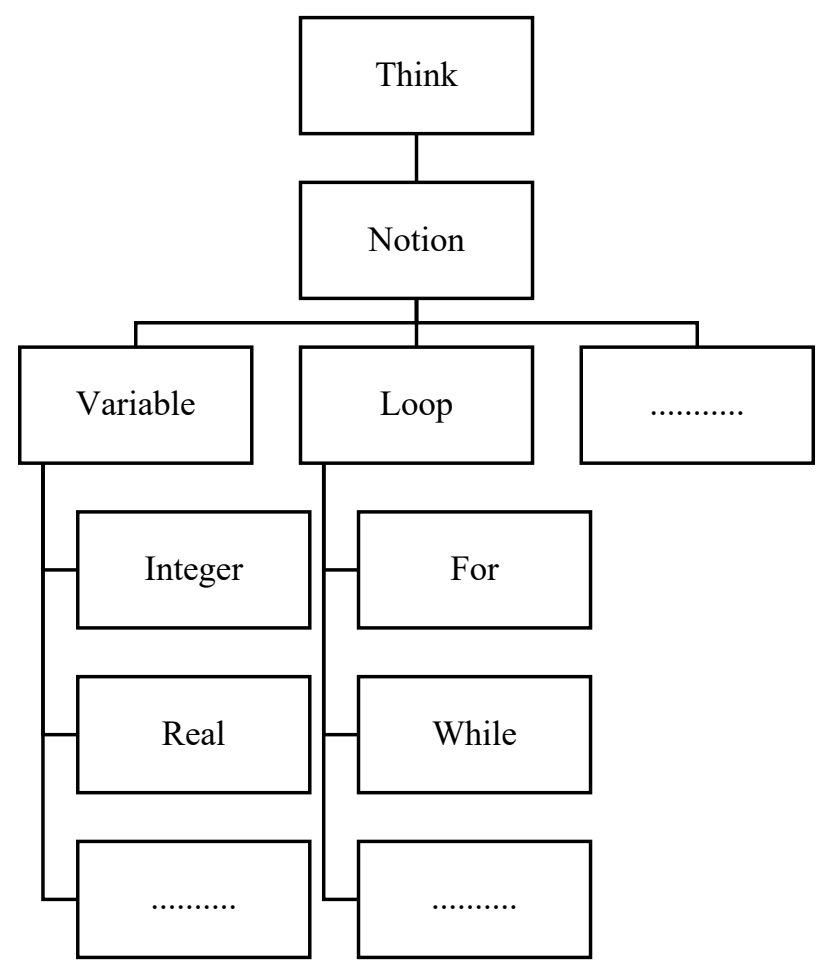

Fig. 2. Excerpt from OntOAlgO

Following Table 1, and in order to detect the relation between the notions, several distances were used:

- $\mathrm{NoA}[\mathrm{Ni}] * \operatorname{NoA}[\mathrm{Nj}]^{*}$ : the relation of the same hierarchical level.

- NoUS [USi] * NoUS [USj]*: the subsumption relation between the NoUS, is a, or $\operatorname{Sim}=1 / 3$ or $2 / 3$ according to the formula of $\mathrm{Wu} \&$ Palmer in the first time to be sure that these notions are closer.

- NoA [Ni] * NoUS [USj]*: the subsumption relation between NoA and NoUS, is a, or $\operatorname{Sim}=2 / 5$ according to the formula of $\mathrm{Wu} \&$ Palmer in the first time to be sure that these notions are closer.

- NoUS [USi] * NoA [Nj] ${ }^{*}$ : the subsumption relation between the NoUS and NoA, is $\mathrm{a}$, or $\mathrm{Sim}=2 / 5$ according to the formula of $\mathrm{Wu} \&$ Palmer in the first time to be sure that these notions are closer. 
Table 1. Adopted Semantic Distance

\begin{tabular}{|l|l|}
\hline \multicolumn{1}{|c|}{ Relation } & \multicolumn{1}{c|}{ Semantic Distance } \\
\hline NoA $[\mathrm{Ni}] *$ NoA $[\mathrm{Nj}]^{*}$ & Equivalent to the same level \\
\hline \multirow{2}{*}{ NoUS [USi] $*$ NoUS $[\mathrm{USj}]^{*}$} & is a \\
\cline { 2 - 3 } NoA $[\mathrm{Ni}] *$ NoUS $[\mathrm{USj}]^{*}$ & Sim $=1 / 3$ or $2 / 3$ according to the Measure of $\mathrm{Wu} \&$ Palmer \\
\hline \multirow{2}{*}{ NoUS [USi] $*$ NoA $[\mathrm{Nj}]^{*}$} & is a \\
\cline { 2 - 2 } & Sim $=2 / 5$ according to the Measure of $\mathrm{Wu} \&$ Palmer \\
\cline { 2 - 2 } & Sim $=2 / 5$ according to the Measure of $\mathrm{Wu} \mathrm{\&} \mathrm{Palmer}$ \\
\hline
\end{tabular}

Step 5: Generate the index: Finally, the index will be generated in an ontology in the form of a semantic network.

Example : Below you will find an example of indexing of an excerpt from an algorithmic LO.

The excerpt: "In the last algorithm, you noticed that a loop was frequently used to increase the value of a variable. It also happens very often that one needs to make a determined number of passages. But, a priori, our structure while does not know in advance how many turns of loop it will perform (it depends on a condition)."

Locating NoA, NoUS, NoUG and the relation between them by the indexing approach: NoA1: "loop", NoA2: "variable", NoA3: "loop" NoUS1: "structure while".

For relations detected are as follows:

Table 2. Semantic Distance Example

\begin{tabular}{|l|l|l|}
\hline Sentene & \multicolumn{1}{|c|}{ Relation } & \multicolumn{1}{|c|}{ Semantic Distance } \\
\hline 1 & $\begin{array}{l}\text { NoA }[\mathrm{Ni}] * \text { NoA }[\mathrm{Nj}]^{*} \\
\text { NoA1 : "loop", NoA2 : "variable" }\end{array}$ & equivalent \\
\hline 2 & $\begin{array}{l}\text { NoUS [USi] * NoA [Nj]*. } \\
\text { NoUS1 : "structure while", NoA3: "loop" }\end{array}$ & is a \\
\hline
\end{tabular}

\section{$4 \quad$ Results and Discussion}

To validate the approach, is applied on algorithmic LO of Christophe Darmangeat and more precisely the chapter on loops which contains more than 2000 words [online]. Available: http://pise.info/algo/

The results of the annotation process are illustrated in the table below where NAS, Number of Annotated Sentences. NASC, Number of Annotated Sentences Correctly. NSAE, Number of Sentences Annotated by the Experts.

Precision is calculated as follows:

Precision $=\frac{\text { NASC }}{\text { NAS }}(2)$

It gives the proportion of relevant documents in relation to noise (non-soliciting documents).

Reminder is calculated as follows:

Recall $=\frac{\text { NASC }}{\text { NSAE }}(3)$ 
It gives the proportion of relevant documents in relation to silence (documents not found)

F-Measure is calculated as follows:

F-Measure $=\frac{2 *(\text { Precision } * \text { Recall })}{\text { Precison }+ \text { Recall }}(4)$

It is a popular measure that combines precision and recall and their weighting.

Table 3. Validation Results

\begin{tabular}{|l|c|c|c|c|c|c|}
\hline \multicolumn{1}{|c|}{ Relation } & NAS & NASC & NSAE & Precision & Recall & F-Measure \\
\hline NoA [Ni] * NoA [Nj]* & 12 & 9 & 14 & 0.7500 & 0.6428 & 0.6922 \\
\hline NoUS [USi] * NoUS [USj]* & 3 & 3 & 8 & 1.0000 & 0.3750 & 0.5454 \\
\hline NoA [Ni] * NoUS [USj] & 2 & 2 & 2 & 1.0000 & 1.0000 & 1.0000 \\
\hline NoUS [USi] * NoA [Nj]* & 4 & 4 & 15 & 1.0000 & 0.2666 & 0.4209 \\
\hline
\end{tabular}

The proposed approach offers important results especially in terms of precision, since indexing is semantic, but it still insufficient, especially when it comes to recall cf. tab.3.

Graph of precision results can be seen in Fig. 3.

Fig. 3 Shows that in a single case there is a different result at 1.000 where there is a repeat of the same NoA that is mistakenly taken as a relation between NoA or there is $\mathrm{a}$ is a lack of meaning or this is due to complicated sentences containing more than one notion however the approach considered relation between NoA otherwise the exate meaning is between other notions.

Graph of recall results can be seen in Fig. 4.

Fig. 4 shows that if the relation between NoA and NoUS is eliminated, there are some gaps. For the relation between NoA the recall is 0.6428 , this is due to repeat of the same NoA that is mistakenly taken as a relation between NoA or this is due to complicated sentences containing more than one notion however the approach considered relation between two NoA but neglects others or the lack of some notions in the ontology because they are short for adopts or is due to the NoUS appear before NoA which disrupts the extraction phase NoUS-NoUS relations are obtained. For the relation between NoUS the recall is 0.3750 there is a limit that is due to the NoA en-

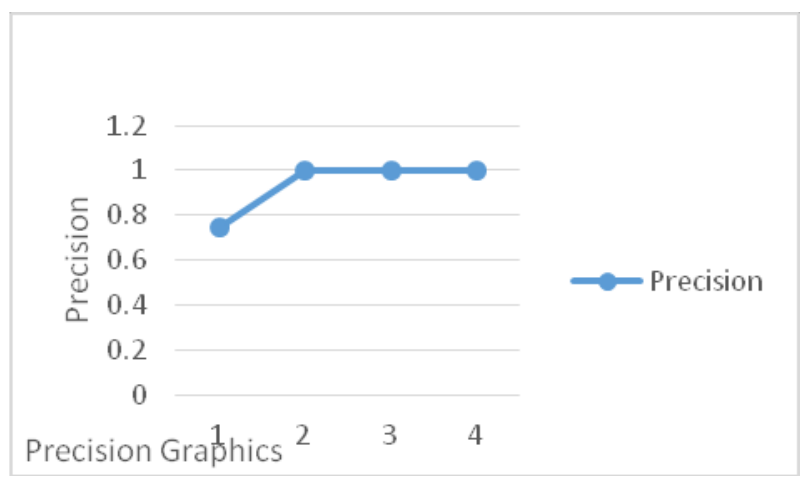

Fig. 3. Graph results precision 


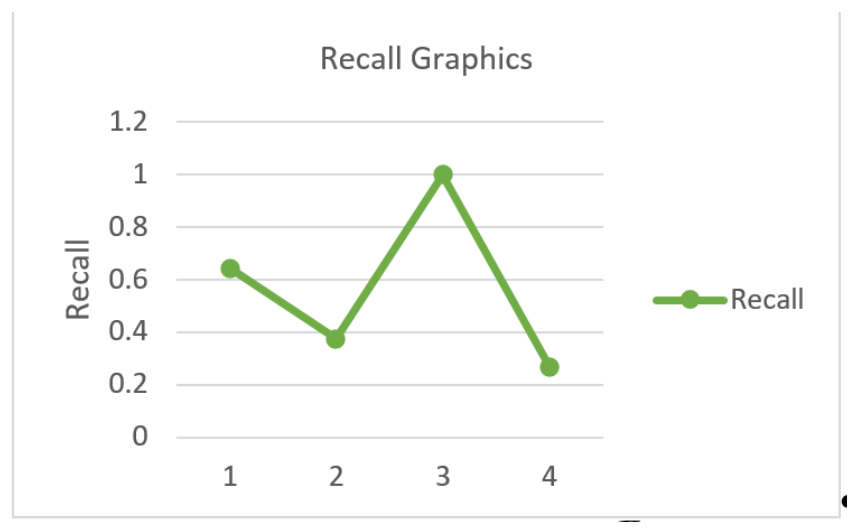

Fig. 4. Graph results recall

counter between NoUS which disrupts the extraction phase and relation type NoUSNoA is obtained or the lack of some notions in the ontology as, for or while, in short. It remains the case of the relation between NoUS and NoA where the reminder is the most cleared in the evaluation 0.2666 , this is due to complicated sentences containing more than a notion however the approach considers the first two notions detected in the sentence or is due to the NoUS encounter between NoUS and NoA which disrupts the extraction phase NoUS-NoUS relations are obtained.

\section{Conclusion}

The presented approach could extract notions deeply in LO according to several categories of sentences fleshing explanations by evaluating notions extracted. The results showed a significant result of precision and recall.

These results are obtained following a consistent approach that is based on semiautomatic metadata and semantic indexing techniques. After the manual identification of the LO and its LOM schema, there is the semi-automatic notions and relation detection with OntOAlgO ontology, and finally the generation of the index.

The notions and their relations will be extracted following linguistic structures and different types of semantic distances allowing to extract deep relations. The approach ensure that the extracted notions convey meaning between them.

As future work, this approach can be expanded to include new type of patterns may include more than two notions; new type of semantic distances and new type of relation such as those between sentences and between paragraphs to extract a more global index on the whole learning object. In addition, the implementation of the approach in MIMTLR is in progress, according to the technologies supported by this engine; J2EE programming language, the JENA API, the RDF language, and the PROTEGE tool. 


\section{References}

[1] Mooers, C.N., Application of Random Codes to the Gathering of Statistical Information, MIT Master's Thesis, 1948.

[2] El Guemmat, K., \& Ouahabi, S. (2018). A Literature Review of Indexing and Searching Techniques Implementation in Educational Search Engines. International Journal of Information and Communication Technology Education (IJICTE),14(2), 7283. https://doi.org/10.4018/IJICTE.2018040106

[3] El Guemmat, K. E., Talea, M., \& Lamrani, E. K. (2015). Implementation and Evaluation of an Indexing Model of Teaching and Learning Resources. Procedia-Social and Behavioral Sciences,191, 1266-1274. https://doi.org/10.1016/j.sbspro.2015.04.654

[4] Achour, H., \& Zouari, M. (2013, June). Multilingual learning objects indexing and retrieving based on ontologies. In Computer and Information Technology (WCCIT), 2013 World Congress on (pp. 1-6). IEEE. https://doi.org/10.1 109/WCCIT.2013.6618710

[5] Biletskiy, Y., Baghi, H., Steele, J., \& Vovk, R. (2012). A rule-based system for hybrid search and delivery of learning objects to learners. Interactive Technology and Smart Education,9(4), 263-279. https://doi.org/10.1108/17415651211284048

[6] Ahmed-Ouamer, R., \& Hammache, A. (2010, October). Ontology-based information retrieval for e-Learning of computer science. In Machine and Web Intelligence (ICMWI), 2010 International Conference on (pp. 250-257). IEEE. https://doi.org/10.1 109/ICMWI.2010.5648113

[7] Wang, S. (2009). Ontology of learning objects repository for pedagogical knowledge sharing. International Journal of Doctoral Studies, 4, 1-12.

[8] Lee, M. C., Tsai, K. H., \& Wang, T. I. (2008). A practical ontology query expansion algorithm for semantic-aware learning objects retrieval.Computers \& Education, 50(4), 1240-1257. https://doi.org/10.1016/j.compedu.2006.12.007

[9] Ghebghoub, O., Abel, M. H., \& Moulin, C. (2008, July). Learning object-indexing tool based on a LOM ontology. In 2008 Eighth IEEE International Conference on Advanced Learning Technologies (pp. 576-578). IEEE. https://doi.org/10.1 109/ICALT.2008.290

[10] Abel, M. H., Benayache, A., Lenne, D., \& Moulin, C. (2007). E-MEMORAe: a content-oriented environment for e-learning. In E-Learning Networked Environments and Architectures (pp. 186-205). Springer London. https://doi.org/10.1007/978-1$\underline{84628-758-97}$

[11] Niwattanakul, S., Eboueya, M., \& Lillis, D. (2006, October). Describing and researching of learning resources with ontology model. In Modern Computing, 2006. JVA'06. IEEE John Vincent Atanasoff 2006 International Symposium on (pp. 214222). IEEE. https://doi.org/10.1109/JVA.2006.15

[12] Dehors, S., \& Zucker, C. F. (2006, July). Reusing learning resources based on semantic web technologies. In Sixth IEEE International Conference on Advanced Learning Technologies (ICALT'06) (pp. 859-863). IEEE. https://doi.org/10.1109/ ICALT.2006.1652578

[13] Pouliquen, B., Le Duff, F., Delamarre, D., Cuggia, M., Mougin, F., \& Le Beux, P. (2005). Managing educational resource in medicine: system design and integration. International Journal of Medical Informatics, 74(2), 201-207. https://doi.org/10.1016/j.ijmedinf.2004.04.020

[14] Smine, B., Faiz, R., \& Desclés, J. P. (2011). A semantic annotation model for indexing and retrieving learning objects. JDIM, 9(4), 159-166. 
[15] Smine, B., Faiz, R., \& Desclés, J. P. (2012, July). Extracting relevant learning objects using a semantic annotation method. In Education and e-Learning Innovations (ICEELI), 2012 International Conference on (pp. 1-6). IEEE. https://doi.org/10.1 109/ICEELI.2012.6360568

[16] Ana Cernea, D., \& Del Moral, E., \& E. Labra Gayo, J. (2008). "SOAF: Semantic Indexing System Based on Collaborative Tagging", Interdisciplinary Journal of E Learning and Learning Objects, Volume 4.

[17] Wu Z., Palmer M., Verbs semantics and lexical selection, in U. A. f. C. L. Stroudsburg, PA (ed.), In Proceedings of the 32nd annual meeting on ACL, volume 2 de ACL '94, p. pp: 133-138, 1994.

[18] Richard K. Belew. Finding Out About: A Cognitive Perspective on Search Engine Technology and the WWW. New York: Cambridge University Press, 2000. Review published in Information Retrieval, Vol. 5, Issue 2-3, April-July 2002.

[19] Amardeilh, F. (2007). Web Sémantique et Informatique Linguisti Linguistique:ions méthodologiques et réalisation d'une plateforme logicielle (Doctoral dissertation, Université de Nanterre-Paris X).

[20] Hassan, S. AND Mihalcea, R. (2009). Learning to identify educational materials. In Proceedings of the Conference on Recent Advances in Natural Language Processing (RANLP).

[21] Valiente, M. C., Sicilia, M. A., Garcia-Barriocanal, E., \& Rajabi, E. (2015). Adopting the metadata approach to improve the search and analysis of educational resources for online learning. Computers in Human Behavior. https://doi.org/10.1 016/j.chb.2014.12.059

[22] Alharbi, A., Henskens, F., \& Hannaford, M. (2012). A Domain-Based Learning Object Search Engine to Support Self-Regulated Learning. International Journal of Computer and Information Technology, 1 (01), 2277-0764.

[23] Al-Omari, M., Al-Omari, M., Carter, J., Carter, J., Chiclana, F., \& Chiclana, F. (2016). A hybrid approach for supporting adaptivity in e-learning environments. The International Journal of Information and Learning Technology, 33(5), 333-348. https://doi.org/10.1108/IJILT-04-2016-0014

[24] Vištica, M., Grubišic, A., \& Žitko, B. (2016). Applying graph sampling methods on student model initialization in intelligent tutoring systems. The International Journal of Information and Learning Technology, 33(4), 202-218. https://doi.org/10.1108/IJILT03-2016-0011

[25] Vuorikari, R., \& Koper, R. (2009). Ecology of social search for learning resources. Campus-Wide Information Systems, 26(4), 272-286. https://doi.org/10.1 $\underline{108 / 10650740910984619}$

[26] Ngom, A. N. (2015). Étude des mesures de similarité sémantique basées sur les arcs. In CORIA(pp.535-544).

\section{Authors}

Dr. Kamal EL Guemmat got his PhD in computer sciences from the Faculty of Sciences Ben M'Sik, Hassan II University. He has several experiences in the field of engineering, teaching, training, and research. He has published some twenty articles in national, internationals conferences and journals. He does his research in Laboratory of Information Processing (LTI) in the Faculty of Sciences Ben M'Sik in which he develops his research in various fields. Also, he does research with other 
groups in different laboratories. His areas of research interest Semantic Indexing, Semantic Web, Information Retrieval Systems, Automatic Processing of Natural Language, Information and Communication Technology for Teaching and Learning, Educational Modeling, E-Learning. Email id:k.elguemmat@gmail.com

Dr. Sara Ouahabi was born in 1988 in Casablanca, Morocco. Took her Bachelor, Master, and PhD degree from Faculty of Sciences Ben M'Sik, Hassan II University. Her area of expertise is teaching, education and research. She develops her research in the Modeling and Information Processing Laboratory (LTIM) in the Faculty of Sciences Ben M'Sik with the collaboration of other research teams. She has participated in several national and international events and conferences and has published her work in international journals. Her interesting area includes Cloud Computing, Distributed Learning, E-learning, Educational Modeling, Semantic Indexing, Information and Communication Technology for Teaching and Learning.

Article submitted 2018-10-19. Resubmitted 2018-11-27. Final acceptance 2018-11-19. Final version published as submitted by the authors. 